\title{
The Intermodal Bike: multi-modal integration of cycling mobility through product and process innovations in bicycle design
}

\author{
Francesca Tosi ${ }^{\mathrm{a},{ }^{*}}$, Alessandro Belli $^{\mathrm{b}}$, Alessandra Rinaldi $^{\mathrm{c}}$, Grazia Tucci $^{\mathrm{d}}$ \\ ${ }^{\mathrm{a}}$ Faculty of Architecture, University of Florence, Via San Niccolò 85, 50125 Florence, Italy \\ ${ }^{\mathrm{b}}$ Tecnologie Urbane Srl, Via Carrand 19, 50133 Florence, Italy, bellimi@dada.it \\ ${ }^{\mathrm{C}}$ Spring Design ${ }^{\circledR}$, Via Capo di Mondo 56, 50136 Florence, Italy, rinaldi@springdesign.net \\ ${ }^{\mathrm{d}}$ Faculty of Architecture, University of Florence, Via Micheli 2, 50100 Florence, Italy, grazia.tucci@unifi.it
}

\begin{abstract}
The paper presents the early results of the UE-FP7 project "The Intermodal Bike". The research aim is to provide a super-compactable, super-lightweight folding bicycle as a realistic solution to graft the cycling mode onto the root of the public or private transportation systems. The folding bikes now on the international market reach weighs between $12-15 \mathrm{~kg}$, with a variable footprint but occupying -when compacted- an average volume of about 100 liters. To encourage the use of this vehicle and to extend it to a larger number of users with different characteristics, the research project has set its goal in increasing as possible compactness and light weight, creating a bicycle with a volume when compacted of 20 liters (reduction factor =5), with a shape of $48 \times 36 \times 12 \mathrm{~cm}$ and a weight of $5 \mathrm{~kg}$. max., ensuring stability and improving vehicle usability and efficiency, during the ride and in the phase of bike folding. To achieve this goal ergonomic and usability tests have been carried out. The tests allowed to find a posture that would ensure efficiency and comfort in the ride to as many users as possible. Parallel tests were made on the vehicle usability in the urban transport system and intermodal. The need for light weight has required special studies on the optimization of the vehicle's architecture and research on super-lightweight materials.
\end{abstract}

Keywords: Design, Sustainable Urban Mobility, Ergonomics, Innovation of Usability, Technological Innovation

\section{Introduction}

The work is driven by the idea that the synergy between cycling and public transportation systems (train, tram, subway, buses and extra-urban) or private (taxi, car)

would increase the share of both modes in the total of urban and suburban trips, and act as an efficiency booster also for the private transportation.

A bicycle is a non - or sparingly- motorized "minimal vehicle", able to handle very efficiently the small scale individual movements, in the range of the short trips typical of the "capillary urban mobility. As efficient as it is, it loses however its appeal on longer commute distances, for a number of reasons. The symbiosis of the bicycle with public or private transportation, more efficient at the medium or long distances, could enhance each mode's strong points.

The synergy between fine grain local mobility and long-haul capacity could lower the access threshold to both cycling and public transport, and make cycling easily and immediately intermodal, with no need of special normative or infrastructural provisions.

Bicycle intermodality could therefore constitute a "third pillar" of incentivation of the cycling mode in the urban mobility (an often stated EU priority in all of its publications on the subject of city mobility)

\footnotetext{
* Corresponding autor. E-mail: francesca.tosi@unifi.it
} 
along with the other two more established instruments at "policy" level (modal incentives and prohibitions) and "infrastructural" level (parking, bike lanes).

The folding bike is the new frontier of the intermodal urban rider.

In leading countries, like Japan, Holland, Great Britain, Belgium, USA, and model cities including London, Paris and Brussels, to name a few, the folding bike is in growing use to get around the city, along with other intermodal public/private transport, as for instance the exchange parking lots. Along with the bike-sharing and bike access to trains and metros, the folding intermodal bike, is an integral part of measures and strategies to address issues and challenges of urban transport problems.

The EU air quality (reduction of $\mathrm{CO} 2$ ) and noise directives require specific actions in urban areas. Improving the urban integration of the bicycle, making it interact with other modes of transport, not only affect the environmental situation, but will also improve accessibility to urban centers, with the prospect of improving their living conditions. Intermodal transport and folding bikes are a practical solution for all situations where it is impossible or difficult to use the classic cycling: for those users who daily flock to urban centers, from the peripheral areas, covering distances that would be difficult to cover with the bike alone, or the hilly towns, where climbs and descents discourage bikers, or urban centers where it is difficult to find bicycle parking, or for those users who live and work in areas not reached in a capillary by means of public transport for those who do not know where to leave their bicycles at night and prefer not to use it, discouraged by the continuing thefts, for those cities where you cannot go on public transport with a city bike, not only because of crowding, but also for the type of platforms that make access impossible. For these and other cases, the folding bike is a real solution. These advantages, if properly communicated, could greatly increase the number of users willing to leave their cars at home and choose to move with a folding bike. For this reason it is very important point to optimize the usability of this medium is an end in itself than in the urban transport system and public / private partnerships, broadening its use to the maximum possible audience, both men and women different age groups.

\section{Ergonomics work package goals: usability, technology and design}

Object of the Ergonomics Work Package of Intermodal Bike is to apply the "Ergonomics for Design" and "User Centered Design" principles to the two areas of methodological research on the Usability of industrial products, and of experimental design:

a) providing all necessary knowledge needed for the configuration of a folding bike characterized by the maximum of comfort and ergonomics;

b) evaluating and optimizing its usability, effective and perceived, in relation to the context of use of the product.

As mentioned above, the latest generation of folding bikes are revolutionizing the way to get around by bike in the great international cities. Equipped with a gearshift up to 6-8 speed, the folding bicycles on the market today weigh between 12 and $15 \mathrm{~kg}$, and fold in an average volume of about 100 cubic decimeters (liters). We ran a survey through a multiple-choice questionnaire on a sample of experienced urban cyclists, found mainly through cyclist associations. In total, 200 respondents were experienced users of different age groups. According to the survey, the folding bikes are considered lightweight and compact enough, but are still perceived by most consumers as strenuous, unreliable and unstable. It 'is therefore necessary to improve not only the lightness and compactness of the vehicle when closed, all elements that would expand its use in general and in particular by weaker users as women, but also improve usability, comfort and pleasure in use, real and perceived.

Regarding task a) the objective was to identify the postural configuration to guarantee maximum pedaling efficiency, protection from stress effects, problems, or damage to the column, neck and arms, even over short distances, the efficacy and in the natural control of the vehicle, with the consequent increase in the perception of comfort and pleasure. The research has focused on aspects other than the traditional ergonomics or physical or related to human factors, extending the work to areas such as anthropometry, biomechanics of posture and movement.

It was useful in particular to identify and analyze user needs especially related to physical factors and consequently to define the requirements of the product and the acceptability thresholds under which the product will be evaluated on an objective basis, that is, referring to quantifiable and measurable metrics. 
The method of ergonomics intervention was aimed at assessing the compatibility between the characteristics of the abilities and characteristics of the product in the usage context, and to ensure and increase both the level of welfare of individuals, that levels performance of the final product. The biomechanics and anthropometry were used as monitoring tools such as posture and information tools that can provide data and evaluation procedures necessary for the approach and the definition of possible design alternatives. Based on the posture of the identified 'ideal', or better than the optimal range of angles of joints involved in cycling, has been designed on the bicycle frame and therefore we have defined an optimal "ergonomic triangle" (handlebar-seat-crank) so as to make the experience of the ride comfortable for the higher percentile of users as possible (from $5^{\circ}$ to $90^{\circ}$ percentile).

Obviously it was necessary to achieve the right balance between moving vehicle usability and its portability when closed. This has led to important decisions that affected the project in general. The choice of a "one size fits many" users, as well as the fact of making a bicycle unisex, and especially the innovative locking system, but does not allow adjustment of the handlebars, unlike for traditional bicycles, made even more important to the study of an ergonomic and comfortable posture for a wide audience.

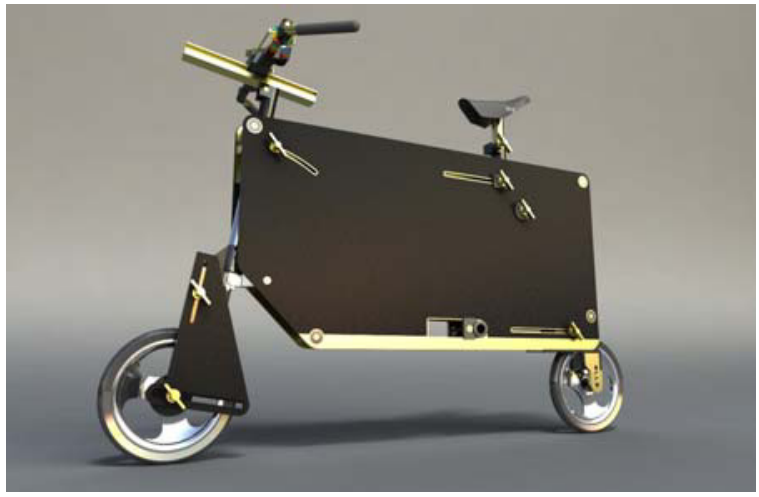

Fig. 1. Measuring model. This prototype has been used to test different postures.
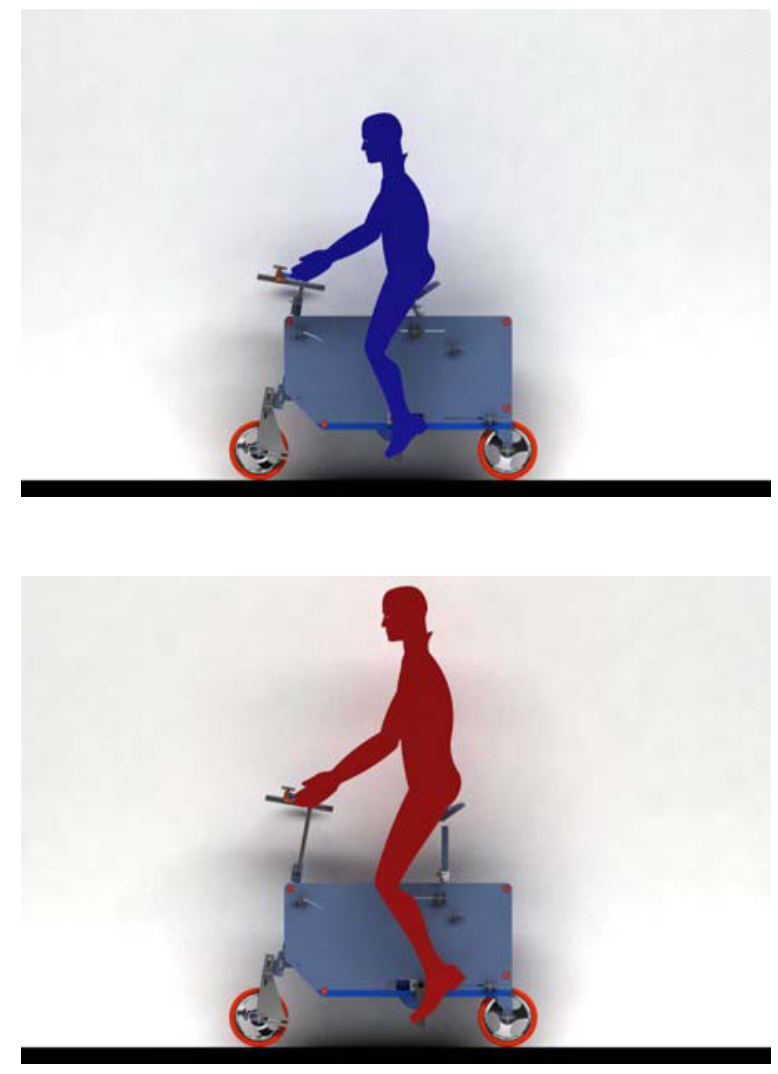

Fig 2-3. Measuring model: all the components are adjustable.

Regarding task b): the objective is to assess the usability of the bicycle in urban intermodal and intermodal transportation by other means, it was decided to include a sample of users, appropriately selected, of some folding bikes already on the market today, and to make empirical tests with them, in collaboration with ATAF, Florence Urban Transport Company, and LPP, Ljubljana transport company, both partners of the project. The tests are currently under development, and are accompanied by interviews and questionnaires and will be completed in autumn 2011. They will conclude with an event of Shared Experience.

The analysis aims to achieve the following:

- deepening knowledge concerning potential users types;

- defining the context and the scenarios of use;

- identifying the problems you might encounter in bicycle use into an inter-modal system with public transport and private;

- defining the different needs between folding bicycle users and classic city bicycle users. The meth- 
od adopted is the direct users observation, developed with people appropriately selected and available to experiment with this type of transportation. Selected users are followed, interviewed and involved in weekly focus groups focused on bike usability.

The tests focus on different aspects of usability, such as time and ease of opening and closing operations, convenience in transportation by bus, tram, train and everything else, comfort perceived by the user in carrying the folded bike, in using to move, etc..

This phase of the research is more related to the User Centred Design. Its aim, in fact, is to create products tailored to the user needs, which use is pleasant and satisfying, and able to guarantee the possibility of carrying out the actions required efficiently and effectively.

\section{Postural and dimensional requirements: survey methodologies}

In order to define dimensional and functional project requirements, two different activities

were carried out in parallel, in order to collect and analyze data related to a selected users group.

The data were collected for cross-checks on the results obtained with the two different methods. The first task called 'People and their Bikes' has resulted in a detection phase of the anthropometric data of "city bikes expert users", or "bicycle as a transportation mean users", appropriately selected according to five different ranges of height, and recognition of their bikes.

Selection is aimed, in particular, to obtain dimensional data of the relationship seat-handlebar-pedal proportional to the user's stature. The analysis was conducted on a sample of 100 users. This phase, together with a questionnaire on the usability of the city bike subjected to the same users, has identified the postures adopted, more or less consciously, by users and considered by them comfortable, and ergonomic.

For the collection of the user's dimensional data to analyzed bicycles was optimized a detection system with 3D digital photogrammetric techniques. The whole process has been automated as much as possible: fully automatic is the camera calibration procedure, as well as the orientation of images (thanks to the use of coded targets).

The application of appropriate markers at strategic points has made it easier the phase of data graphic representation.
It was thus possible to represent point by point the geometry of each bike, each of which have been defined in three-dimensional coordinates. In particular, the points were found according to the relationship seat-handlebar-crank center, the center of the handlebar grips and the distance between the wheels. To verify the accuracy of acquisition method, were also made sample manual measurements. The collected data have high accuracy, with a tolerance of about + $10 \mathrm{~mm}$.
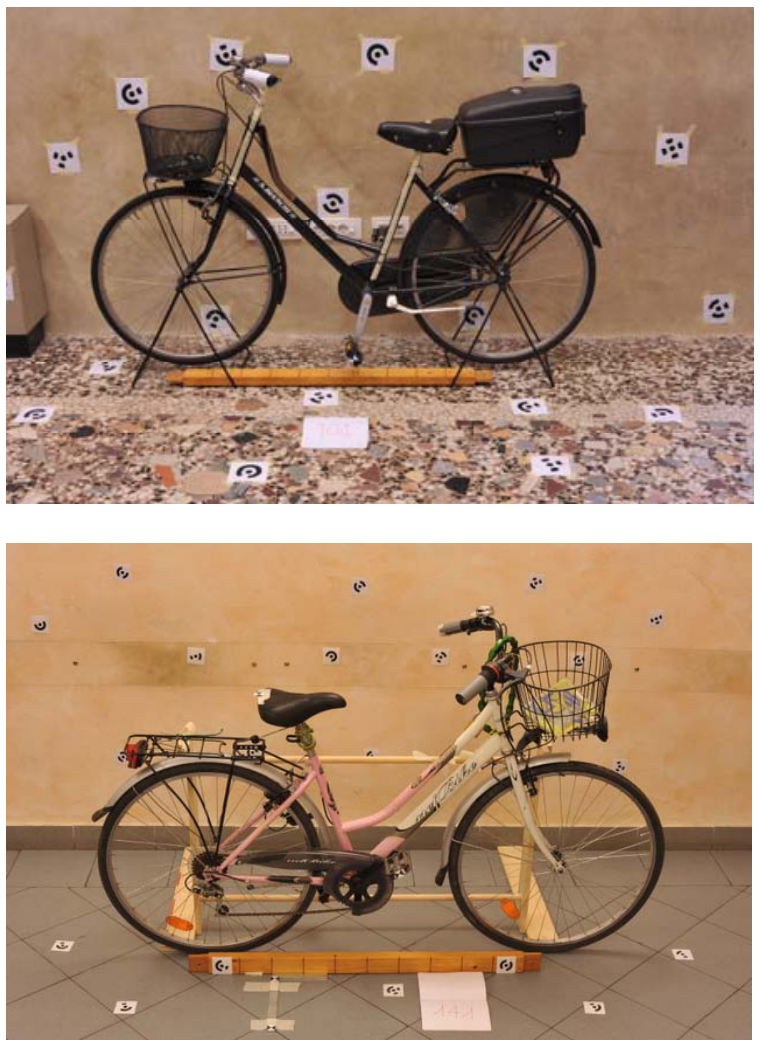

Fig. 4-5. Bicyles during the detection phase

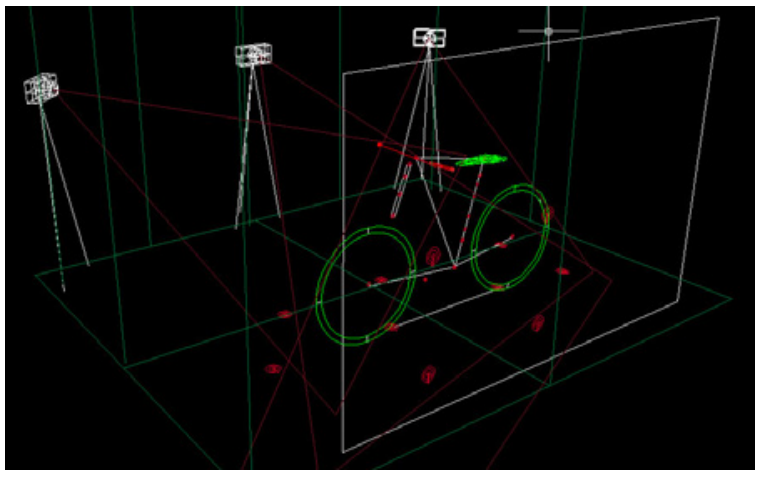



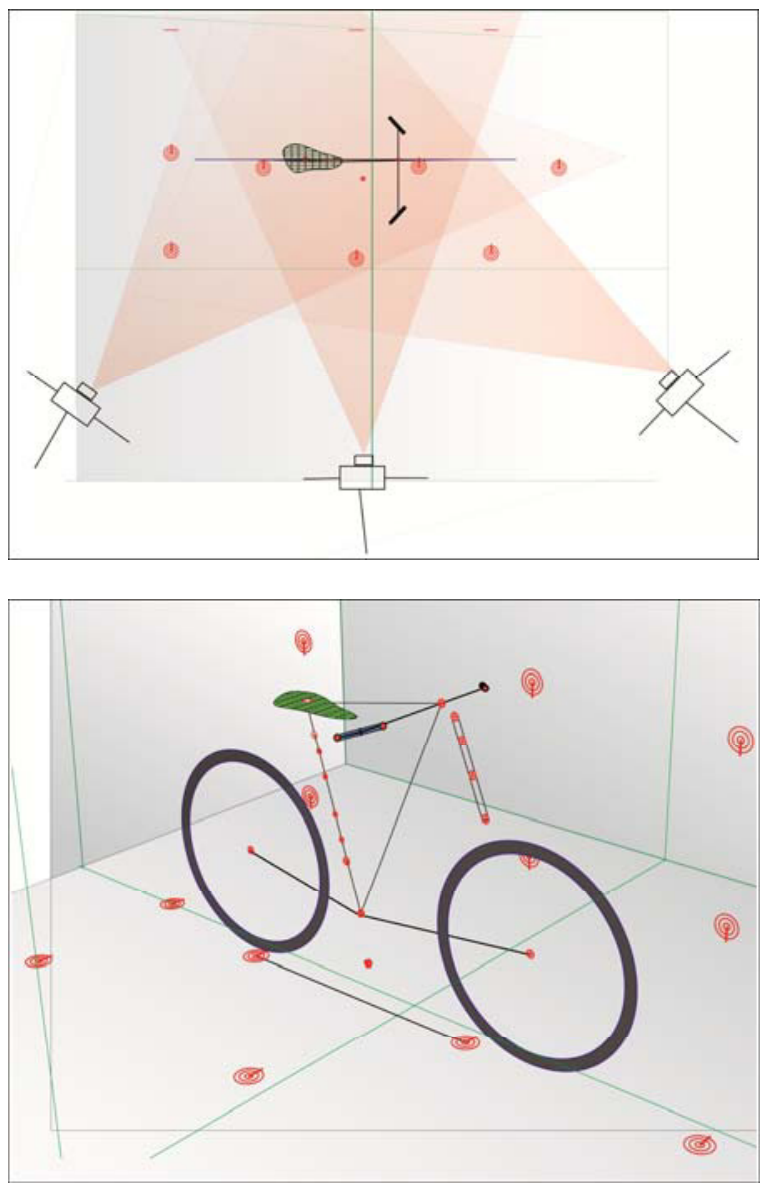

Fig. 6-7-8. 3D CAD processing of bicycles with digital photogrammetric system

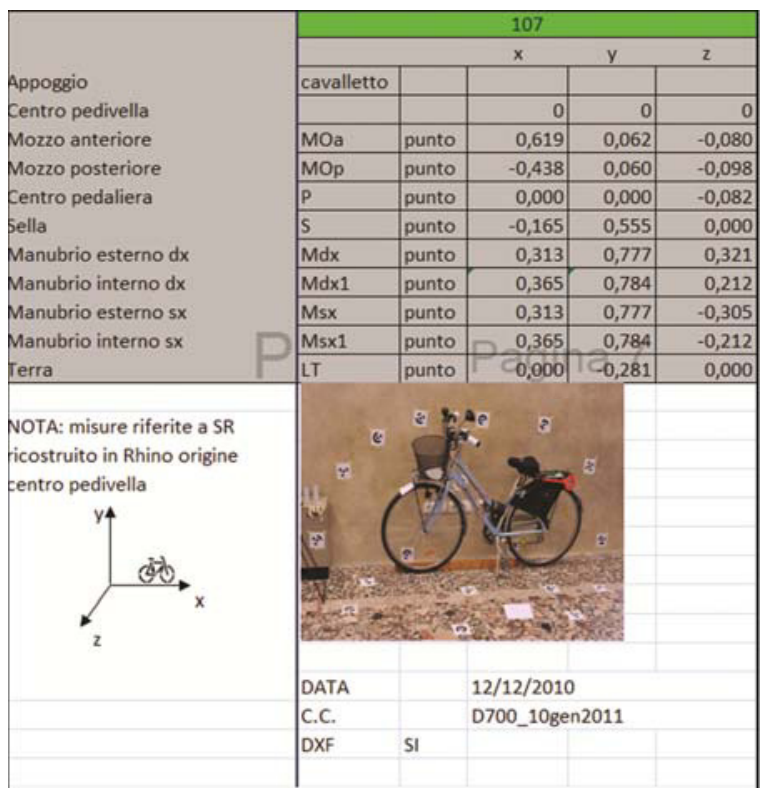

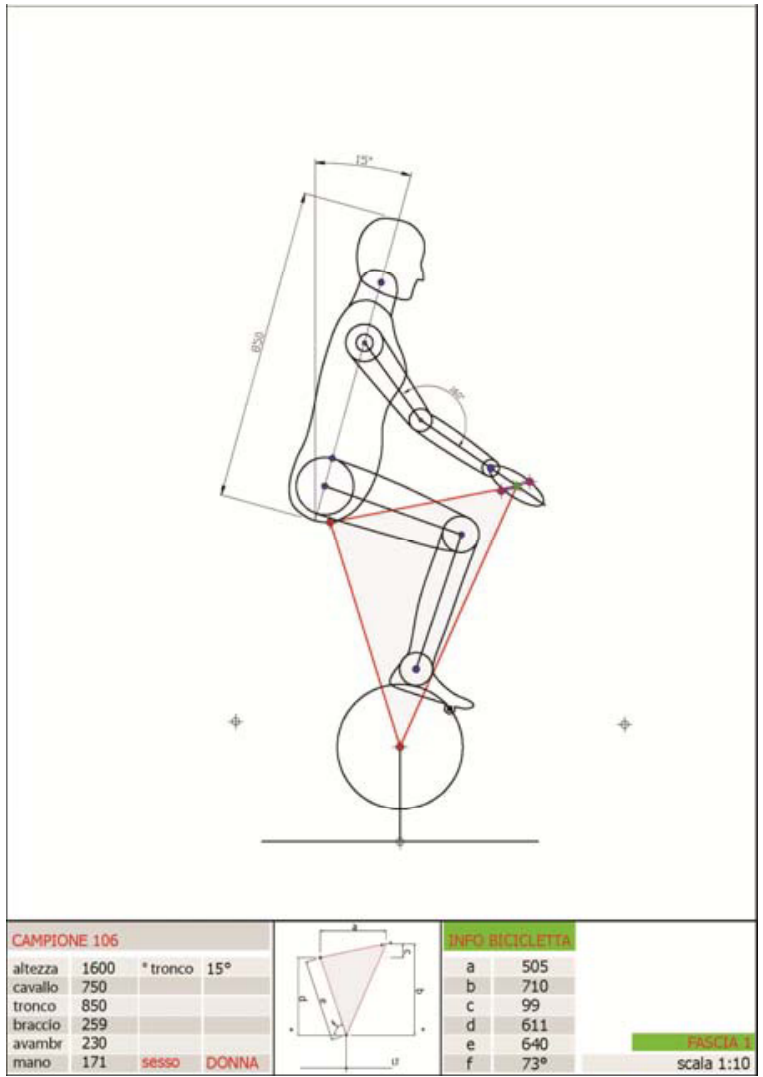

Fig. 9-10. The data collected

A second research task "Biomechanical analysis of posture and movement", developed by of Occupational Medicine research group of University of Florence (Vincenzo Cuppeli, Giulio Arcangeli, Marco Petranelli) is currently under completion.

The data processed by our work group will be compared and integrated with those developed by occupational medicine group, in order to define an integrate the evaluation of usability and biomechanics aspects.

\section{Ergonomics and technology innovation: design for light weight}

The obvious thing to say about making something very lightweight, is that it is necessary to put "less stuff" in it. In other words a lightweight object is also a more efficient object in the use of the materials.

There are several ways to reduce the material 
keeping constant the performance, depending on the relative importance of the structure's stiffness and ultimate strength. That is if it's more important to control how much deformation a structure can have, or if the deformation is less important than the ability of the structure to absorb a lot of stress and rebound with no broken elements.

A bicycle falls in the first case. It must be designed for stiffness, as a "wobbly" frame feels insecure, makes the ride uncomfortable because the rider must use part of his or her energy to check continuously the posture, and the elastic work of the frame absorbs more rider energy dissipating it.

An ideal bicycle has a very stiff frame, moving to accurately chosen areas, outside a closed "hard cell", the desired elastic elements for comfort and suspension. This happens for instance in all carbon-fiber modern frames.

The case of a "collapsing" bike frame is particularly difficult because the necessary joints break the material continuity of "monolithic" normal bike frames. The most common solution has been traditionally an "open" frame based on a large tube able to resist large bending and torcional stresses, that is the loading that any material has more difficulty dealing with. This translates directly into very heavy bicycles, as such tubes must necessarily have very robust sections.

Our project had the "light weight" as one of the two central requirements, and therefore we chose to follow a path common in bridge and large scale constructions but very seldom used in the bike industry: the space-frame, and to be more precise the pretensioned space frame.

A space-frame does not rely on torsion and bending to resist the external loads, but rather uses only "pure" tension and compression stresses, that is the stresses that any material has less problems with. The space-frame, and especially the type with pretensioned members -often termed, in certain cases, "tensegrity systems"- are in absolute terms the structures that have the least material per volume occupied or surface covered.

In our case the space-frame is particularly suited to realize an opening-closing structure, as a collection of rods and cables, all very similar in length, lends itself to be packed in a very dense configuration, with a minimum of "air" in between, obtaining therefore a very compact pack.

Ultimately the bike frame is a sort of very stiff "cage" that offers the support to the cycling components as pedals, seat etc. but with a form that is quite different from the classic "diamond-frame". In partic- ular the seat-regulation system doesn't have a "seat tube", but can be designed to follow -within a certain range- a move on a line not bound to the frame. This helps in the design of a one-fits-many bicycle that must be adapted to bodies of different build.

\section{Conclusions}

- The ergonomic study is pointing in the direction of a "corrected Dutch" posture as the most suitable for non-sport, daily urban commute, of the type the users of an "intermodal bike" are expected to do.

- "Corrected" refers to some early results of the field tests, pointing to a tendency to lean forward more as the subjects grow tired in the test effort. Discovering this tendency has been useful in the calibration of the "reference posture", given the peculiar frame and seat adjustment of Bike Intermodal.

- The particular frame, where most members have similar shapes and dimensions, allows for an automatic, spring-loaded opening-closing system.

- The materials used to build the frame are mostly steel, aluminum and thermoplastic composites.

\section{References}

[1] A.A.V.V., Due ruote per il futuro. Prima Conferenza Nazionale della Bicicletta, Ediciclo, Portogruaro (VE), 2008.

[2] R. Buckminster Fuller, Tensegrity, Portfolio and Art News Annual N 4, 1961.

[3] O. Frei, Finding Form: towards an architecture of the minimal, Axel Menges, Stuttgart, 1996.

[4] E. Heartney and K. Snelson, Forces made visible, Hudson Hills Press, Manchester VT USA, 2009.

[5] Libro verde dell'Unione Europea, Verso una nuova cultura della mobilità urbana, 2007.

[6] J. Mapes, Pedaling revolution: how cyclist are changing American Cities, Oregon State University Press-Corvallis, 209.

[7] R. Motro, Tensegrity: structural systems for the future, Hermes Science Publishing, London, 2003.

[8] Prima Conferenza Nazionale della Bicicletta, Due ruote per il futuro, Ediciclo, Portogruaro (VE), 2008.

[9] A. R. Tilley, The measure of man and woman: human factors design,

[10] S. Pheasant, Body space, Anthropometry, Ergonomics and the Design of Work, Taylor \& Francis, Londra, 1997 